\title{
Validez y confiabilidad de la escala de actitudes hacia el reciclaje y uso responsable de papel en los estudiantes de la UNMSM
}

Validity and reliability of the scale of attitudes towards the recycling and responsible use of paper in students of the UNMSM

\author{
Gaby Vargas Vargas \\ Universidad Nacional Mayor de San Marcos, Lima Perú \\ Contacto: gvargasv@unmsm.edu.pe \\ https://orcid.org/0000-0002-4383-3200
}

\begin{abstract}
Resumen
El método del estudio fue psicométrico, porque se aplicó un conjunto de procedimientos que permitieron valorar cuantitativamente características psicológicas como la validez y confiabilidad de la escala de actitudes hacia el reciclaje y uso responsable de papel. Asimismo, la investigación tuvo como propósito elaborar la escala de actitudes hacia el uso responsable de papel, en los estudiantes de la UNMSM. Hecho que ubicó el estudio como investigación tecnológica, cuyo producto fue la obtención del cuestionario mencionado; el método utilizado para tal fin fue el descriptivo correlacional, con diseño transversal, porque los datos se obtuvieron en un solo momento (Hernández y otros 2001). La población estuvo constituida por 241 estudiantes. El diseño muestral fue probabilístico y su tamaño se calculó con el procedimiento de Cochrane al 0,05 de error y a un nivel de confianza del 0,95 , requiriendo una muestra de 241 estudiantes para cubrir las exigencias mencionadas. Igualmente, el diseño de la investigación fue de tipo no experimental de corte transeccional, porque los datos se obtuvieron en un solo momento. También podemos mencionar que el estudio fue de tipo aplicado porque permitió confrontar teorías planteadas con la realidad. Además, la técnica utilizada fue la prueba y el instrumento de recolección de datos la escala de actitudes hacia el reciclaje y uso responsable de papel. La técnica utilizada corresponde a los fundamentos de la psicometría para elaborar cuestionarios que en el estudio sirvieron para medir las actitudes hacia el reciclaje y uso responsable de papel. Teóricamente se utilizó la teoría de Fishbein y Ajzen (1975), como orientadores conceptuales. Para demostrar la validez, se utilizó el método de constructo utilizando la consistencia interna de los factores y test total. Para la confiabilidad, se empleó el método de la consistencia interna ítem test total y el método de las mitades, en el primer caso mediante la fórmula de Crombach y en el segundo con la fórmula de Spearman. El criterio de normalización fueron percentilares y la puntuación escalar T.
\end{abstract}

Palabras clave: confiabilidad, validez, instrumento, actitudes, reciclaje, uso de papel, responsabilidad social. 


\begin{abstract}
The method of the study was psychometric because it was applied a set of procedures that allowed quantitative evaluation of psychological characteristics such as the validity and reliability of the scale of attitudes toward recycling and responsible use of paper. Likewise, the research aimed to elaborate the scale of attitudes towards responsible use of paper in students of the UNMSM. Done that I locate the study as technological research, whose product was the obtaining of the mentioned questionnaire; The method used for this purpose was the descriptive Correlational, with cross-sectional design, because the data were obtained in a single moment (Hernández \& Others 2001). The population consisted of 241 students. The sample design was probabilistic whose size was calculated with the Cochrane procedure at 0.05 of error and at a confidence level of 0.95 , requiring a sample of 241 students to cover the mentioned requirements.

Also the design of the research was of non-experimental type of transactional cut, because the data were obtained in a single moment. We can also mention that the study was applied type because it allowed to confront theories raised with reality. In addition the technique used was the test and the instrument of data collection the scale of attitudes toward recycling and responsible use of paper. The technique used corresponds to the fundamentals of psychometry to elaborate questionnaires that in the study was to measure attitudes towards recycling and responsible use of paper theoretically used the theory of Fishbein \& Ajzen (1975), as conceptual guides. To prove the validity, the construct method was used using the internal consistency of the factors and total test. For reliability, we used the internal consistency test item total test and the method of the halves, in the first case using the Crombach formula and in the second with the Spearman formula. The normalization criteria were percentile scores and scalar T.
\end{abstract}

Keywords: reliability, validity, instrument, attitudes, recycling, paper use, social responsability.

\title{
Introducción
}

En nuestro medio, la construcción y validación de escalas de medición en relación con las actitudes ambientales es limitada, no existen escalas con características de nuestra población porque la mayoría de instrumentos de medición han sido elaborados en otros países, obviamente considerando características culturales diferentes a nuestra población; ello ha permitido realizar la propuesta de la construcción y validación de la escala de actitudes ambientales hacia el reciclado y uso responsable de papel con características propias de la población de estudio de la Facultad de Letras de la Universidad Nacional Mayor de San Marcos. En el 
ámbito universitario, desde el enfoque de la responsabilidad social, es importante contar con instrumentos de recolección de datos que reúnan criterios de validez y confiabilidad para poder aplicar y obtener información precisa con relación a las actitudes ambientales y de esta manera tomar decisiones para promover buenas prácticas en beneficio del desarrollo sostenible.

\section{Metodología}

El estudio demostró la validez y confiabilidad del instrumento de actitudes hacia el reciclaje y uso responsable de papel en la población de estudiantes de la Universidad Nacional Mayor de San Marcos. En el estudio se observó que el constructo no es unidimensional debido al número de factores encontrados en hallazgos similares a otros estudios, evidenciando la existencia entre 3 y 6 factores. Los especialistas de la psicometría Thurstone (1952), Anastasi y Urbina (1998), Nunnally (1987), Thordike y Hagen (1970) afirmaron que los test, al igual que los instrumentos de medición, deben demostrar que miden lo que se proponen, de modo que la teoría de fondo oriente para encontrar la unidad de medición que sirva como referencia; estas unidades deben responder a la definición de lo que se quiere medir, el objeto no solo debe ser identificado sino necesariamente debe contar con una definición. Por ello fue necesario realizar el análisis de validez de contenido para poder determinar si el instrumento evalúa y logra alcanzar su objetivo. Si precisamos los resultados presentados en la tabla 1 en el sentido de que los valores chi cuadrado resultan significativos al 0.01 de margen de error, se verá que el cuestionario elaborado tiene validez de contenido.

Por otro lado se analizó la consistencia interna de cada una de las áreas del cuestionario y del test total, lo que permitió verificar el postulado teórico. Así, los coeficientes entre áreas son significativos pero no mayores de 0.50 , lo que significa que sus ítems corresponden a cada área diferencialmente. Esta afirmación se sustenta debido que los coeficientes entre cada área y el puntaje total de la prueba presentan valores mayores de 0.50 .

Asimismo la confiabilidad desde el punto de vista de Anastasi y Urbina (1998), Thordike y Hagen (1970), Nunnally (1987), Thurstone (1952), quienes 
se refirieron al error de medida, quiere decir que los instrumentos de medición si bien no son exactos, deben propender a disminuir al máximo la poca confiabilidad aumentando los índices de fiabilidad. Es importante considerar las fuentes de error relacionadas con el propio examinado, al que aplica la medición y al propio instrumento; por ello se debe tener en cuenta, a la hora de aplicación, el rapport del examinado, la familiaridad del examinador con el instrumento, el compromiso con el factor que mide, así como que el instrumento esté adaptado a las condiciones y lenguaje de los examinados.

También el análisis de confiabilidad por consistencia interna pudo determinar que los coeficientes de Crombach van desde un mínimo de 0.70 y cuando se eliminaron los ítems al que pertenece en la fila correspondiente se registró hasta un máximo de 0.77. Estos resultados nos dicen que los ítems del cuestionario corresponden a un mismo concepto cuya confiabilidad máxima ha sido de 0.77 . Además, para verificar la confiabilidad de cada una de las áreas se ha efectuado el mismo análisis encontrando valores alfa para cada una de las áreas que van desde un mínimo de 0.78 hasta un máximo de 0.81 . Ello confirma que el cuestionario elaborado mide las actitudes hacia el reciclaje y uso responsable de papel dentro en los márgenes de error permitidos

Por otra parte, para verificar si los resultados obtenidos con las puntuaciones observadas de los ítems impares correspondían a los ítems se aplicó el análisis de confiabilidad por el método de las mitades, prueba que permitió calificar tres puntuaciones: para los ítems impares, pares y para la prueba total. Asimismo, se ha utilizado la prueba de Spearman para la estimación de los coeficientes de correlación habiendo encontrado un coeficiente de 0.849 y para la extensión total de la prueba coeficiente similar 0.849 , los mismos que indican que el cuestionario elaborado mide con el mínimo de riesgo de error. Al obtener la raíz cuadrada de este coeficiente tenemos el valor Rox que explicita el índice de confiabilidad que es de 0.9214 o del 92.14\%, dejando entrever que el índice de no confiabilidad es solo del 7.86\%; por tanto, nos encontramos con un cuestionario que mide actitudes hacia el reciclaje y el uso responsable de papel con un margen de error permitido y deseable. 
En consecuencia la normalización se refiere a criterios más o menos estables de interpretación de los resultados, para lo cual los puntajes directos se convierten en puntajes escalares que hacen posible la comparación entre una prueba, que en este caso corresponde entre un área, y la otra que para tal fin se utilizó. Por la extensión de los ítems de cada área y la calificación, corresponde una escala de 5 grados; de esta forma, mayor puntaje significa mayor aceptación de los criterios o de los indicadores, obteniendo así el puntaje bruto o directo. Estos puntajes han sido convertidos a eneatipos que son escalas donde el puntaje escalar máximo es 9 y el puntaje escalar mínimo es 1, siendo la media escalar 4.5. Así, con estos datos hemos convertido los puntajes directos a eneatipos. Además, utilizando los puntajes totales hemos creído conveniente elaborar un baremo percentilar de forma que a través del puntaje directo podemos ubicar en qué valor del 1 al 100 se encuentra el examinado en cuanto a actitudes hacia el reciclaje y uso responsable de papel.

\section{Resultados}

A continuación presentamos los resultados de análisis de validez y confiabilidad para la escala de actitudes hacia el reciclaje y uso responsable de papel.

\section{Análisis de contenido de ítems}

En la tabla 1 se presentan los resultados de contenidos de los ítems analizados con el método de opinión de expertos y contrastado con valores de chi cuadrado, donde los valores son válidos y se demuestra la homogeneidad de ítems. 
Tabla 1

Análisis de contenido de ítems por el método de opinión de expertos contrastado con la prueba de valores chi cuadrado

\begin{tabular}{|c|c|c|c|c|}
\hline Ítem & $\mathrm{FO}$ & FT & $\mathrm{Ji2}$ & Decisión \\
\hline 1 & 100 & 50 & $81 * *$ & Válido \\
\hline 2 & 100 & 50 & $81 * *$ & Válido \\
\hline 3 & 100 & 50 & $81 * *$ & Válido \\
\hline 4 & 100 & 50 & $81 * *$ & Válido \\
\hline 5 & 100 & 50 & $81 * *$ & Válido \\
\hline 6 & 100 & 50 & $81 * *$ & Válido \\
\hline 7 & 100 & 50 & $81 * *$ & Válido \\
\hline 8 & 100 & 50 & $81 * *$ & Válido \\
\hline 9 & 100 & 50 & $81 * *$ & Válido \\
\hline 10 & 100 & 50 & $81 * *$ & Válido \\
\hline 11 & 100 & 50 & $81 * *$ & Válido \\
\hline 12 & 100 & 50 & $81 * *$ & Válido \\
\hline 13 & 100 & 50 & $81 * *$ & Válido \\
\hline 14 & 100 & 50 & $81 * *$ & Válido \\
\hline 15 & 100 & 50 & $81 * *$ & Válido \\
\hline 16 & 100 & 50 & $81 * *$ & Válido \\
\hline 17 & 100 & 50 & $81 * *$ & Válido \\
\hline 18 & 100 & 50 & $81 * *$ & Válido \\
\hline 19 & 100 & 50 & $81 * *$ & Válido \\
\hline 20 & 100 & 50 & $81 * *$ & Válido \\
\hline
\end{tabular}

$(* *) \mathrm{P}<0.01$ 


\section{Análisis del coeficiente de correlación ítem / total}

Se analizó los coeficientes de correlación de cada una de las áreas del cuestionario con cada una de ellas y con el test total; ello con el propósito de verificar si se tratan de áreas independientes o, por lo contrario, si pueden fusionarse o desaparecer. El resultado ha confirmado el postulado teórico, los coeficientes entre áreas son significativos pero no mayores de 0.50 ; esto significa que sus ítems corresponden a cada área diferencialmente, así, esta afirmación se sustenta debido que los coeficientes entre cada área y el puntaje total de la prueba con excepción de una sola área (moralidad) han registrado coeficientes mayores de 0.50 tal como se puede leer en la tabla 2 .

Tabla 2

Validez de ítems por el método de constructo: coeficientes de correlación ítem test total

\begin{tabular}{|c|c|c|c|c|c|c|c|}
\hline Ítem & To G & İtem & To G & Ítem & To G & İtem & To $\mathrm{G}$ \\
\hline 1 & 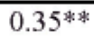 & 7 & 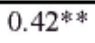 & 13 & 0.44 *** & 19 & 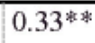 \\
\hline 2 & $0.33^{* *}$ & 8 & $0.44 * *$ & 14 & $0.47 * *$ & 20 & $0.34 * *$ \\
\hline 3 & 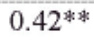 & 9 & $0.44^{*}$ * & 15 & 0.38 ** & 21 & $0.35^{*}$ * \\
\hline 4 & $0.48^{*}$ ** & 10 & $0.43^{\text {*2* }}$ & 16 & $0.38^{* * *}$ & 22 & $0.34^{*}$ *水 \\
\hline 5 & $0.49 * *$ & 11 & $0.45 * *$ & 17 & $0.40 * *$ & 23 & $0.31 * *$ \\
\hline
\end{tabular}

(**) $\mathrm{P}<0.01$

Tabla 3

Validez de ítems por el método de constructo: coeficientes de correlación ítem test total

\begin{tabular}{rcccccc}
\hline & Conocimiento & isposición & Hábitos & Valoración & Interés & $\begin{array}{r}\text { Total } \\
\text { general }\end{array}$ \\
\hline Conocimiento & 1.00 & & & & & \\
Disposición & $0.33^{* *}$ & 1.00 & & & & \\
Hábitos & $0.21^{* *}$ & $0.35^{* *}$ & 1.00 & & & \\
Valoración & $0.12^{*}$ & $0.21^{* *}$ & $0.34^{* *}$ & 1.00 & & \\
Interés & $0.13^{*}$ & $0.19^{* *}$ & $0.21^{* *}$ & $0.31^{* *}$ & 1.00 & \\
Total general & $\mathbf{0 . 5 9 * *}^{* *}$ & $\mathbf{0 . 5 9 ^ { * * }}$ & $\mathbf{0 . 6 1}^{* *}$ & $\mathbf{0 . 6 1}^{* *}$ & $\mathbf{0 . 5 4}^{* *}$ & $\mathbf{1 . 0 0}$ \\
\hline
\end{tabular}

(*) $\mathrm{P}<0.05$

(**) $\mathrm{P}<0.01$ 


\section{Análisis de confiabilidad por consistencia interna}

Para demostrar que el instrumento posee la propiedad psicométrica de confiabilidad se ha aplicado el método de análisis de consistencia interna, el mismo que ha sido estimado con el alfa de Crombach, observando en la tabla 4 correlación en las puntuaciones. Es decir, el instrumento presenta consistencia en las puntuaciones que forman el constructo teórico que se desea medir.

\section{Tabla 4}

Coeficiente de confiabilidad por el método de la consistencia interna Ítem test total utilizando el coeficiente de Crombach, con una muestra de 381 examinados

\begin{tabular}{|c|c|c|c|c|}
\hline Estadísticos & $\begin{array}{c}\text { Media de } \\
\text { la escala si } \\
\text { se elimina } \\
\text { el elemento }\end{array}$ & $\begin{array}{c}\text { Varianza } \\
\text { de la escala } \\
\text { si se } \\
\text { elimina el } \\
\text { elemento }\end{array}$ & $\begin{array}{l}\text { Correlación } \\
\text { elemento- } \\
\text { total } \\
\text { corregida }\end{array}$ & $\begin{array}{c}\text { Alfa de } \\
\text { Crombach } \\
\text { si se } \\
\text { elimina el } \\
\text { elemento }\end{array}$ \\
\hline \multicolumn{5}{|l|}{ Ítems } \\
\hline Conocimiento: 1 & 166.22 & 597.241 & .308 & .705 \\
\hline Conocimiento: 2 & 166.36 & 598.105 & .289 & .706 \\
\hline Conocimiento: 3 & 166.53 & 593.139 & .384 & .703 \\
\hline Conocimiento: 4 & 166.68 & 590.609 & .444 & .701 \\
\hline Disposición: 5 & 166.79 & 588.240 & .453 & .700 \\
\hline Disposición :6 & 166.78 & 592.988 & .376 & .703 \\
\hline Disposición :7 & 166.80 & 591.274 & .377 & .702 \\
\hline Disposición :8 & 166.81 & 590.326 & .444 & .709 \\
\hline Hábitos: 8 & 166.78 & 591.169 & .391 & .702 \\
\hline Hábitos: 10 & 166.72 & 590.504 & .400 & .702 \\
\hline Hábitos: 11 & 166.74 & 592.694 & .382 & .703 \\
\hline Hábitos: 12 & 166.55 & 590.756 & .567 & .702 \\
\hline Valoración: 13 & 166.77 & 588.273 & .400 & .701 \\
\hline Valoración: 14 & 166.85 & 589.345 & .415 & .701 \\
\hline Valoración: 15 & 166.94 & 589.880 & .401 & .702 \\
\hline Valoración: 16 & 166.56 & 589.770 & .405 & .703 \\
\hline Interés: 17 & 166.95 & 587.366 & .424 & .700 \\
\hline Interés: 18 & 167.02 & 593.497 & .334 & .704 \\
\hline Interés: 19 & 166.91 & 593.036 & .332 & .704 \\
\hline Interés: 20 & 167.89 & 586.055 & .445 & .703 \\
\hline
\end{tabular}


Los resultados de la tabla 5 permiten observar la consistencia en las puntuaciones del total de la escala y de cada una de sus dimensiones, obteniendo resultados entre el rango de aceptable buena y buena.

Tabla 5

Resumen de coeficientes de confiabilidad por el método de consistencia interna de Alfa de Crombach

\begin{tabular}{lll}
\hline & $\begin{array}{l}\text { Alfa de } \\
\text { Crombach }\end{array}$ & Decisión \\
\hline Conocimiento & 0.78 & Aceptable buena \\
Disposición & 0.80 & Buena \\
Interacción & 0.80 & Buena \\
Hábitos & 0.81 & Buena \\
Valoración & 0.81 & Buena \\
Interés & 0.79 & Aceptable buena \\
Total General & 0.77 & Aceptable buena \\
\hline
\end{tabular}

\section{Análisis de confiabilidad por el método de las mitades}

En la tabla 6 se presentan los resultados del análisis en dos partes, de modo que se tuvo el mismo número de ítems y fueron consideradas paralelas, garantizando de esta manera la equivalencia.

Tabla 6

Coeficiente de correlación por el método de las mitades utilizando la fórmula de Spearman-Brown

\begin{tabular}{lll}
\hline \multicolumn{3}{l}{ Correlación entre formas } \\
\hline Coeficiente de & Longitud igual & .849 \\
Spearman-Brown & Longitud desigual & .849 \\
\hline $\mathrm{P}$ & & .006 \\
\hline
\end{tabular}




\section{Discusión}

La educación ambiental es uno de los retos para las instituciones de educación superior, por ello es importante abordar el estudio de las actitudes ecosostenibles porque permiten predecir el tipo de motivación que dirige al sujeto a estar preocupado por el medio ambiente (Weigel y Weigel, 1978). En tal sentido, el estudio de las actitudes como la predisposición aprendida, no innata, estable y que puede cambiar, a reaccionar de una manera valorativa favorable o desfavorable, ante un individuo, grupos, ideas, situaciones, etc., es importante para el campo de la educación ambiental. Así, el estudio de las actitudes específicas de los estudiantes de la Universidad Nacional Mayor de San Marcos con relación al reciclaje y uso responsable de papel, se constituye en una variable intermedia entre el estímulo (el objeto de la actitud) y la respuesta o manifestación externa de alguna manera mensurable. El aporte científico del estudio es que permite contar con un instrumento de medición con características de la población de estudio y enfocado en la realidad de nuestro país; a nivel educativo la aplicación de la prueba permitirá recoger datos de fuente directa y que ayuden en la solución de problemas y toma de decisiones en relación con el medio ambiente.

Por lo tanto, los resultados obtenidos han demostrado la confiabilidad y validez del instrumento con características propias de la población de estudio; además, la validez del instrumento diseñado para una aproximación del entendimiento de las actitudes y su aplicación práctica orientada a contribuir en las líneas de investigación de la educación ambiental.

\section{Referencias bibliográficas}

Anastasi, A. y Urbina, S. (1998). Test psicológicos. Ciudad de México, México: Pretince Hall.

Fishbein, M., y Ajzen, I. (1975). Belief, Attitude, Intention, and Behavior: An Introduction to Theory and Research. Reading, EE. UU.: Addison-Wesley.

Hernández, B., Corral, V., Hess, S. y Suárez, E. (2001). Sistemas de creencias ambientales: un análisis multimuestra de estructuras factoriales [Environmental belief systems: A multisample covariance analysis of factor structures]. Estudios de Psicología, 22, 53-64. 
Nunnally, J. (1987). Teoría psicométrica. Ciudad de México, México: Mc Graw Hill.

Thordike, L. y Hagen, E. (1970). Medición en psicología y educación. Ciudad de México, México: Trillas.

Thurstone, L. L. (1952). L. L. Thurstone. En G. Lindzey (ed.), A History of Psychology in Autobiography, vol. 4 (pp. 294-321). Englewood Cliffs, EE. UU.: Prentice Hall.

Weigel, R. y Weigel, J. (1978). Environmental concern: The development of a measure. Environment and Behavior, 10(1), 3-15. doi: $10.1177 / 001391657810100$. 